\title{
Oportunidades y desafíos de los docentes en programas de biología con asignaturas teórico-prácticas de dos instituciones colombianas de educación superior bajo restricciones de pandemia
}

\author{
Giovany Guevara ${ }^{1}$ (D) \\ Jorge Enrique García-Melo ${ }^{2}$ (D) \\ Lida Marcela Franco-Pérez ${ }^{2}$ (D) \\ ${ }^{1}$ Universidad del Tolima, Colombia. ${ }^{2}$ Universidad de Ibagué, Colombia
}

Resumen. Desde el inicio de la pandemia por COVID-19, las instituciones académicas de todo el mundo se vieron obligadas a cerrar sus campus y ajustar su enseñanza a un modelo de educación a distancia. Una de las actividades de mayor dificultad estuvo relacionada con los programas académicos que involucran prácticas de laboratorio o de campo, las cuales no pueden cubrirse adecuadamente utilizando solo computadoras, videos o acciones demostrativas. A pesar de estas restricciones, por parte de los docentes se adaptaron o desarrollaron propuestas alternativas, como el uso de nuevas fuentes de enseñanza; sin embargo, no se puede pensar en ellas sin considerar sus implicaciones en el aprendizaje de los estudiantes modernos: nativos digitales, más participativos y con deseos de fortalecer sus futuras competencias prácticas. Mediante el uso de un laboratorio en casa que integra computadora, muestras en vivo, cámara digital y herramientas remotas, y plataformas en línea, describimos las experiencias de la enseñanza-aprendizaje en línea durante la pandemia actual, en la Universidad de Ibagué y la Universidad del Tolima en Ibagué, Colombia. Después de la retroalimentación, destacamos las oportunidades y los principales problemas, inquietudes, y desafíos futuros tanto para docentes como estudiantes de pregrado de los programas de biología, para continuar bajo una enseñanza y aprendizaje híbridos, durante la nueva era normal o postpandemia en educación superior, sin descuidar el aseguramiento de la calidad.

Palabras clave: biología; Colombia; coronavirus; educación superior; teletrabajo.

Oportunidades e desafios dos professores em programas de biologia com disciplinas teórico-práticas de duas instituições de ensino superior colombianas sob restrições pandêmicas

Resumo. Desde o início da pandemia do COVID-19, as instituições acadêmicas de todo o mundo foram obrigadas a fechar seus campi e ajustar seu ensino a um modelo de educação a distância. Uma das atividades mais difíceis estava relacionada a programas acadêmicos que envolvem práticas de laboratório ou de campo, que não podem ser adequadamente cobertas usando apenas computadores, vídeos ou ações demonstrativas. Apesar dessas restrições, os professores adaptaram ou desenvolveram propostas alternativas, como a utilização de novas fontes de ensino; no entanto, não se pode pensar nelas sem considerar suas implicações para a aprendizagem dos alunos modernos: nativos digitais, mais participativos e desejosos de fortalecer suas competências práticas futuras. Através do uso de um laboratório doméstico que integra computador, amostras ao vivo, câmera digital e ferramentas remotas e plataformas on-line, descrevemos as experiências de ensino-aprendizagem on-line durante a atual pandemia, na Universidade de Ibagué e na Universidade de Tolima, em Ibagué , Colômbia. Após o feedback, destacamos as oportunidades e os principais problemas, preocupações e desafios futuros para professores como para alunos de graduação dos cursos de biologia, para continuarem no ensino e aprendizagem híbridos, durante a nova era normal ou pós-pandemia no ensino superior, sem negligenciar a garantia de qualidade.

Palavras-chave: biologia; Colômbia; coronavírus; ensino superior; teletrabalho.

Opportunities and challenges for teachers in biology programs with theoretical-practical subjects from two Colombian institutions of higher education under pandemic restrictions Abstract. From beginning the COVID-19 pandemic, academic institutions worldwide were forced to close their campuses and adjust their teaching to a distance education model. One of such tasks of major difficulty was related to academic programs that involve fieldwork or lab practices, which cannot be adequately cover by using only computers, videos, or demonstrative actions. Despite these concerns some adjusts, or new alternative proposals were developed by the teachers, such as the use of new teaching sources, however those cannot be thought of without consideration of their learning implications on the modern students: digital natives, more participatory and wishing to strengthen their further practical skills. By using an at-home lab integrating computer, in vivo samples, digital camera, and remote tools and online platforms, we described the experiences of teaching-learning online during the current pandemic, at the Universidad de Ibagué and Universidad del Tolima in Ibagué, 
Colombia. After feedback we highlighted the opportunities and the main issues, concerns, and further challenges in both teachers and undergraduate students of biology programs for continuing under a hybrid teaching and learning during new normal age or post-pandemic in higher education without neglecting quality assurance.

Keywords: biology; Colombia; coronavirus; higher education; telework.

\section{Introducción}

El primer caso de coronavirus fue reportado oficialmente en Wuhan (China) el 31 diciembre 2019. El 11 marzo 2020 la organización mundial de la salud-OMS declaró a la COVID-19 como una pandemia, con más de 118000 casos en más de 110 países y territorios alrededor del mundo, bajo una amenaza creciente de una diseminación global (Jandrić, 2020). A partir de ese momento los gobiernos de los distintos países respondieron de formas radicalmente diferentes, aun sin tener claro qué medidas efectivas adoptar (e.g., cierre de fronteras, autoaislamiento, distanciamiento social y/o cuarentena). A partir de marzo 2020 las escuelas, colegios, universidades, y centros de investigación del mundo, iniciaron la transición rápida o paulatina hacia una interacción educativa en línea (Ibarra-Caicedo, 2021a; Melo-Becerra et al., 2021). Para las familias, los niños y los estudiantes en aislamiento propio o distanciamiento social, la oportunidad de completar con éxito ese año académico fue muy importante. Sin embargo, ese logro estuvo asociado con condiciones de mucho estrés tanto para ellos y sus familias como para los docentes (Digión y Álvarez, 2021; Miguel, 2020; Moral et al., 2021).

Algunas universidades -que ya operaban virtualmente-, programas académicos y docentes (principalmente los más jóvenes) estaban relativamente bien preparados para la enseñanza y el fomento de la investigación en línea; otros, trabajaban en las noches y los fines de semana en el desarrollo de material audiovisual para disponerlo de manera remota, contando con el apoyo de sus estudiantes y colegas (Cipagauta, 2021). En otros casos, debido a la carga de trabajo que se generó, algunos docentes diseñaron y grabaron sesiones de clase para luego compartirlas con los estudiantes, como una forma de cumplir lo mejor posible con el desarrollo completo de las asignaturas. Finalmente, muchos académicos lograron esa transición de manera rápida, soportados por las herramientas virtuales disponibles en el momento, la familiarización con nuevos programas computacionales y por la gran cantidad de material libre ya existente en línea (videos, infografías, laboratorios virtuales). Aquellos que enseñaban de forma individual y contextual se enfrentaron a distintos desafíos (Jandrić et al., 2020), como es el caso de los docentes vinculados a formación posgradual.

Uno de los desafíos de los docentes para la formación integral de sus alumnos en las instituciones de educación superior (IES) con programas presenciales, es lograr y mantener una propuesta didáctica innovadora para realizar actividades en asignaturas de corte prácticas $(P)$, teórico-prácticas $(T / P)$ o T/P que involucran al menos una salida de campo (fuera del aula de clase/laboratorio o del campus universitario). A pesar de que la inclusión de prácticas virtuales complementarias, ya habían sido advertidas en experiencias educativas previas (Barberá y Badia, 2005; De la Sen y Díaz, 2009), esta situación fue mucho más evidente cuando surgió la necesidad de realizar dichas prácticas en el contexto de la pandemia por COVID-19 (Argel et al., 2020; Vargas-Straube et al., 2021). Desde hace ya varios años, en el entorno virtual o 
remoto, se desarrollaba en universidades con sus programas, capacitaciones en línea (cursos por video chat, webinars, podcasts), repositorios de libre acceso, entre otros. Lo que no había ocurrido, es que millones de personas a nivel mundial, utilizaran las herramientas de la Internet y las redes sociales al mismo tiempo y para los mismos propósitos (Cevallos et al., 2021; Giraldo et al., 2021; Moreira et al., 2020).

Entre los otros desafíos asociados con la pandemia y aquellos que persistirán por un buen tiempo, se encuentra el hecho de que los alumnos que cursan asignaturas virtuales deben tener una serie de conocimientos previos y de énfasis (dependiendo de si se trata de una asignatura obligatoria u electiva), algunos de ellos marcados por la huella o vacíos potenciales que ha dejado esta nueva modalidad de enseñanzaaprendizaje, a los que se vieron enfrentados por al menos tres periodos académicos (2020 y 2021). Asimismo, desde el punto de vista del docente se prevé un manejo de las dificultades para poner en contacto a los alumnos con procesos que se realizan de forma real en las empresas e industrias o que directamente guardan realización con su futuro profesional (De la Sen y Díaz, 2009).

En el presente documento, se destacan algunas experiencias colombianas relacionadas con las vivencias de docentes de asignaturas teórico-prácticas (T/P) y T/P con salidas de campo de los programas presenciales de Biología Ambiental y de Biología en la Universidad de Ibagué (UI, institución privada) y la Universidad del Tolima (UT, institución pública), respectivamente, durante el inicio y parte de la dinámica de la pandemia por COVID-19 (marzo 2020 - octubre 2021). Aunque se hace énfasis en la asignatura Zoología, dados los resultados exitosos, esta experiencia se puede aplicar a otras asignaturas de corte similar. Asimismo, se destacan otros elementos que se asocian con las oportunidades de mejora tanto para estudiantes como docentes, dependiendo de las restricciones o no, relacionadas con el acceso a equipos e Internet y bajo la expectativa de continuar en una enseñanza-aprendizaje mediada por una educación híbrida durante la postpandemia, que promueva o fortalezca el aseguramiento de la calidad educativa.

\section{Revelación de las brechas digitales ( $\approx$ desigualdad social)}

Un factor crítico tanto en las IES públicas como privadas colombianas de orden regional y aun en las de orden nacional, es la identificación y reconocimiento de las brechas digitales, principalmente en el uso y acceso de tecnologías de la información y la comunicación (TIC). Sin embargo, se trata de una situación generalizada en la mayoría de los países latinoamericanos, lo cual se evidenció aún más durante la pandemia. Se confirmaron algunos elementos de desigualdad social, relacionados con las condiciones para acceder a la educación virtual, dependiendo entre otras cosas, de la ubicación geográfica, recursos económicos y tecnológicos, y capacitación y experiencia en el uso de TIC (Gómez-Arteta y Escobar-Mamani, 2021; Miguel, 2020). Además de la falta de preparación de la comunidad educativa (profesores, alumnos, directivos, padres de familia, etc.), se sumó la falta de respuesta oportuna del sistema educativo y de los gobiernos en general, que pusieron al descubierto muchas falencias, incluso de aquellos que ya estaban familiarizados con ellas (analfabetismo digital), las cuales hicieron que las brechas digitales desde los ámbitos de acceso, de uso y 
de competencia en TIC, se profundizaran durante la crisis por el coronavirus SARSCoV-2, amenazando las opciones y opacando los intereses de los sujetos educativos (Gómez-Arteta y Escobar-Mamani, 2021; Villela y Contreras, 2021).

En el caso del acceso, conocimiento y capacitación en uso de TIC, la mayor parte de la población en áreas urbanas tiene mejores oportunidades de uso de la Internet, comparado con las zonas rurales. Al igual que otros países latinoamericanos, un bajo porcentaje de los estudiantes residentes rurales cuenta con Internet en casa bajo las restricciones de la pandemia. Por lo tanto, durante esta situación se exacerbaron aún más las fuertes desigualdades, según la condición socioeconómica de los estudiantes (Bercheñi y Mariño, 2021; Ordorika, 2020; Ruiz-Aquino, 2020). Sin embargo, la transformación digital exigida a partir de la COVID-19 contribuyó a fortalecer el conocimiento y capacitación acelerada u "obligada", principalmente, en profesores y estudiantes, adaptando tanto sus espacios de trabajo como la reprogramación de las velocidades de la Internet y el repotenciamiento o cambio de sus equipos computacionales; estos dispositivos representan una herramienta indispensable para estudiar y trabajar, en un mundo cada vez más interconectado.

De una u otra forma, cada una de estas acciones que voluntaria o involuntariamente se generaron a raíz de la necesidad de seguir con el proceso de enseñanzaaprendizaje, bajo distintos niveles de conectividad, permitieron hasta cierto punto, una disminución de la brecha digital en todos los sectores, al generar más espacios para actividades que antes eran impensables o estaban subestimadas en presencialidad. Igualmente aportó a la consolidación de tecnologías basadas en inteligencia artificial, al crecimiento de la infraestructura de los países y al mejoramiento de plataformas digitales y aplicaciones móviles (Núñez-Cortés, 2021). En general, la crisis de la COVID-19 mostró que el acceso a la tecnología es fundamental para una educación remota, y resaltó la necesidad urgente de un esfuerzo nacional para cerrar estas brechas (Vargas et al., 2020).

En las universidades colombianas analizadas, una privada (https://www. unibague.edu.co/) y una pública (https://www.ut.edu.co/), se crearon alternativas para apoyar a los estudiantes de escasos recursos que presentaban algún impedimento o que pertenecían principalmente a estratos socioeconómicos bajos. En ese sentido, ambas universidades fortalecieron el acceso a becas, fomentaron convenios con empresas proveedoras del servicio de Internet, además de varios incentivos económicos (bingos solidarios, no cobro por concepto de matrícula) o el suministro de equipos (tabletas equipadas con tarjetas sim y plan de datos) para evitar la deserción y garantizar el mejor desempeño académico posible. A pesar de los esfuerzos compartidos, algunas restricciones continuaron, marcadas por falta de empleo de los padres o la redistribución de los ingresos para subsanar necesidades vitales como alimentación y salud.

A continuación, se resaltan algunos de los elementos que generaron oportunidades de mejora y desafíos para el aseguramiento de la calidad en las IES seleccionadas, tomando como referencia el análisis vivencial de docentes del área de zoología. 


\section{Clases rutinarias, proyectos de investigación y tutorías en trabajos de grado}

A pesar de que muchas universidades trataron de mantener el proceso de admisión sin mayores inconvenientes, desde la declaratoria de pandemia, el inicio de labores estuvo siempre supeditado a la dinámica del contagio y cuarentenas reiteradas; y sobre el desarrollo de los acontecimientos, las universidades se enfocaron en la planificación y construcción de sus clases remotas basadas en entornos virtuales (Figueroa et al., 2021).

En las clases rutinarias de los programas académicos; es decir, aquellas asignaturas de corte presencial que se vieron avocadas a una transición a la virtualidad se desarrollaron nuevos enfoques, mayor énfasis en el trabajo asincrónico apoyado por videos complementarios o explicativos, documentos dirigidos (artículos, capítulos de libro), guías de laboratorio virtual, reorganización de las asignaturas, entre otros. Los profesores y estudiantes tuvieron capacitaciones constantes en herramientas remotas, manejo de información en repositorios, estrategias para los exámenes y prácticas de laboratorio, y fortalezas en entornos virtuales. En el caso de estudiantes que iniciaban su proyecto de investigación asociado con su trabajo de grado, el cual incluía actividades de laboratorio y/o campo, se vieron seriamente rezagados. Para estos estudiantes, se enfatiza en la necesidad de utilizar las bases de datos electrónicas suscritas por la IES, para temas actuales y revisión de artículos clásicos, de tal manera que se pudiera contar con un documento lo más completo posible, hasta cuando se pudiera retomar la actividad de campo o de laboratorio. En otros casos, algunos estudiantes optaron por cambiar de modalidad de grado, analizar información previamente colectada o suspender el semestre académico mientras podían retomar la experimentación y colecta de datos. En el caso del Programa de Biología, la Universidad del Tolima avaló la graduación a través de presentación y defensa de una Monografía; es decir, una revisión exhaustiva sobre un tema de interés biológico, ecológico o ambiental.

A medida que se fueron levantando las restricciones y se avaló el regreso paulatino a las universidades, las asignaturas que ya habían demostrado desarrollos adecuados de sus contenidos, mediante el uso de varias de las estrategias mencionadas anteriormente junto con videos demostrativos y en 3D, retomaron actividades de laboratorio con prácticas experimentales demostrativas en el formato tradicional (Argel et al., 2020) vs. prácticas demostrativas en pandemia o alternancia, en ambas universidades (UI, UT). Durante el tiempo de alternancia, y aun desde cuando se levantó parcialmente la cuarentena, se privilegiaron los espacios verdes como los jardines botánicos, bosques urbanos, y colecciones biológicas (museos, herbarios) para el desarrollo de asignaturas (T/P) o T/P con salidas de campo, frente a la dificultad de realizar actividades fuera del campus y toma de datos por al menos casi dos años. En cualquier caso, se exigían los elementos de protección personal, y se mantenían otros protocolos de bioseguridad como el distanciamiento social y el aforo en los espacios más concurridos. 


\section{Asignaturas teórico-prácticas (T/P) y $T / P$ con salidas de campo}

En áreas de estudio como biología, química, física, psicología, idiomas, enfermería, medicina y otras profesiones afines, las necesidades de enseñanza enfatizan la importancia de desarrollar aspectos teóricos y prácticos (T/P). Buena parte de estas asignaturas incluyen actividades con prácticas de laboratorio, y varias de ellas son T/P con salidas de campo. Al desarrollar los aspectos prácticos (tanto en laboratorio como en campo), se da especial énfasis a las actividades que enseñan a los estudiantes métodos experimentales, cómo sintetizar observaciones y una gama de habilidades comunicativas y prácticas que se vieron completamente limitadas durante el confinamiento y se extendieron durante todo el aprendizaje remoto (Gamage et al., 2020).

Aunque la interacción virtual funciona bien para la construcción de conocimiento a través de la entrega de contenido y la supervisión de algunos procesos, tiene limitaciones para desarrollar las habilidades prácticas en laboratorio y en campo. Por ejemplo, para llevar a cabo un laboratorio de la asignatura Zoología (integrada, de invertebrados, o de vertebrados), para el Programa de Biología o Biología Ambiental, es necesario utilizar ejemplares de organismos que se encuentran en colecciones biológicas de docencia o científicas, o mediante la colecta de organismos en campo durante las prácticas. Sin embargo, el aprendizaje remoto comenzó a limitar las competencias de los alumnos, ya que es casi que imposible sustituir el carácter inmersivo y realista de las actividades prácticas. Por tanto, se plantearon varios desafíos de cómo el docente universitario puede generar alternativas, especialmente cuando se cuenta con equipos y con la experticia audiovisual (Figura1), que permitan interactuar con el alumno de manera práctica, bajo un enfoque virtual.

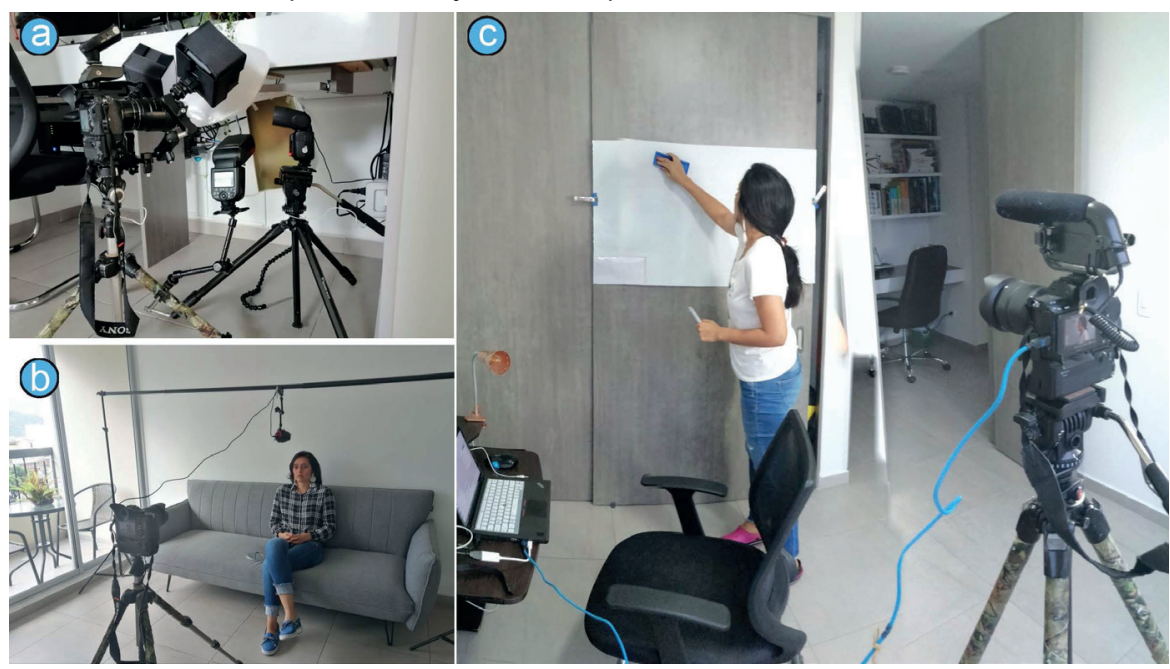

Figura 1. Organización del área de trabajo en casa para el desarrollo de asignaturas teóricas o teórico-practicas. a) Equipos con cámara fotográfica, trípodes y flashes para transmisión en vivo, b) montaje para realización de clases pregrabadas o interacción en vivo, c) disposición de una pizarra, cámara grabando y conectividad con el computador y alguna herramienta de interacción (e.g., Cisco Webex®, Facebook Live ${ }^{\circledR}$, Google Meet ${ }^{\circledR}$, Microsoft Teams $\AA$, YouTube ${ }^{\circledR}$, Zoom $\left.\AA\right)$. 
Para estos laboratorios virtuales, se utilizaron herramientas de simulación y realidad aumentada, de acceso libre en línea con especímenes en 3D (e.g., https://3dlabs.upm.es/web/laboratorios.php?idioma=es). Los laboratorios remotos permiten la realización de experimentos a través de la Internet, mientras que las actividades basadas en vídeo proporcionan una descripción general paso a paso de un laboratorio real para que los estudiantes puedan visualizar todo el proceso experimental y su entorno a través de un video que transmite el profesor desde su espacio de trabajo adaptado en su casa, utilizando fauna urbana o especímenes adquiridos en el supermercado. El diseño del laboratorio permitió a los estudiantes realizar experimentos que incorporaban el aprendizaje autónomo, interactivo y colaborativo en anatomía externa e interna (Figura 2).

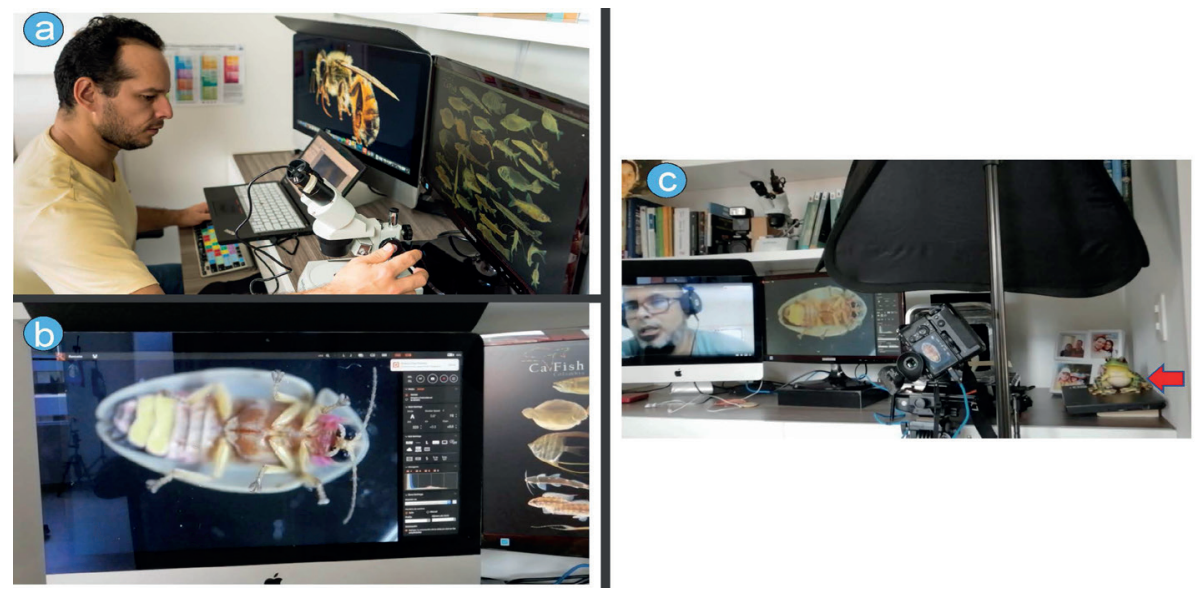

Figura 2. Área de trabajo en casa para la interacción sincrónica con estudiantes de la asignatura T/P Zoología del Programa de Biología Ambiental de la Universidad de Ibagué (Tolima, Colombia). a) montaje con cámara acoplada a un estereomicroscopio, computadores y muestra de invertebrado, b) imagen del invertebrado para compartir con los estudiantes visto en el computador de enlace, c) montaje final para realizar la sesión durante la clase programada. La flecha indica uno de los organismos (en plástico) utilizados en la clase, cuando no se dispone de muestras biológicas.

A pesar de que la recepción de estas metodologías por parte de los alumnos fue positiva en su mayoría, los vacíos que se pueden percibir en estudiantes de Biología, es altamente variable, hasta el punto de que un estudiante de cuarto semestre aún no ha podido tener un contacto físico y menos una experticia para manejar idóneamente un microscopio o un estereomicroscopio. Además, existe una gran limitación, que está asociada con las destrezas de documentación científica que pueda tener el profesor y el tiempo de preparación de las clases. Por ejemplo, durante 2020 y parte de 2021, las clases prácticas del laboratorio fueron preparadas utilizando estas aproximaciones, pero la habilidad técnica para los montajes (Figura 2) y los recursos necesarios pueden llegar a ser muy altos, y en buena parte de los casos, supone un gran desgaste físico y mental para el profesor.

La otra limitación está relacionada con la importancia de la cultura y el medio ambiente en las experiencias prácticas de aprendizaje de los estudiantes; no obstante, cuando disminuyeron las limitaciones de acceso al campus y el retorno gradual, progresivo 
y seguro a la nueva normalidad mediante clases de laboratorio presenciales y remotas, permitió, durante la alternancia, encuentros accidentales, fortuitos y casuales que mejoraron lazos con compañeros y el aprendizaje se hizo más agradable. Por lo tanto, fue importante la cohesión grupal, la confianza, el respeto y el adecuado trabajo en grupo. Sin embargo, estos enfoques "innovadores" necesitan de una gestión tecnológica amplia (Figuras 1 y 2) con la cual no cuentan la mayoría de las universidades, ni los docentes.

\section{Desarrollo de competencias y resultados de aprendizaje}

Existe una discusión generalizada, de un lado por los docentes que orientamos asignaturas T/P y, por otro, por los estudiantes que las cursaron bajo las restricciones impuestas por la pandemia, relacionada con el hecho de que las competencias, principalmente aquellas del "saber-hacer" no se lograron incorporar por al menos 1.5 semestres académicos; es decir, para un set tripartita de varias asignaturas. Por lo tanto, a priori se juzga que el nivel de dichas competencias y los mismos resultados de aprendizaje se asocian con un detrimento en la calidad de la formación de los futuros profesionales biólogos. Asimismo, se crea anticipadamente una atmósfera de dificultades para la dominancia de ciertos temas, especialmente para los interesados en una formación posgradual. Esta situación es más compleja cuando se considera que varias asignaturas T/P del pensum en ambas universidades, no contó con la fortaleza de las salidas o prácticas de campo asociadas. Tanto en la UI como en la UT, durante el periodo 2020 y primer semestre de 2021, no se contó con la posibilidad de realizar prácticas académicas fuera de sus campus. Solo en pocos casos, y hacia el final de 2021 se lograron consolidar algunas salidas a entornos verdes urbanos o rurales cercanos; sin embargo, se mantuvieron las restricciones de aforo, distanciamiento social, uso continuo de los elementos de protección personal, entre otros protocolos de bioseguridad (e.g., organización de los grupos para ingreso a los vehículos y transporte mismo).

\section{Retorno seguro al campus: alternancia y reinicio de la presencialidad}

Se ha observado que la crisis de la COVID-19 ha tenido y tendrá un impacto importante en la inscripción de estudiantes nacionales e internacionales, con efectos negativos especialmente en las IES privadas (Ordorika, 2020; Santana-Sardi et al., 2020). Varios estudiantes iniciaron su semestre, pero no lo pudieron culminar de manera exitosa. Entre otros aspectos, por la situación de acceso a equipos y conectividad permanente. Después de superar las restricciones internacionales, nacionales, regionales y locales, apoyadas por el sostenimiento de los protocolos de bioseguridad, aun con buena parte de la comunidad universitaria con el esquema de vacunación completo (una o dos dosis más de refuerzo), se plantearon las opciones de retorno progresivo al campus principal para adelantar actividades de docencia, inicialmente en asignaturas teóricas y T/P con opciones para desarrollar prácticas de laboratorio demostrativas. Las universidades realizaron llamados frecuentes a través de consultas o formularios en línea (Figura 3), donde se incluyeron preguntas relacionadas con la situación de salud del personal y la disposición que tuviera el docente para retomar las actividades cotidianas. Sin embargo, allí sobresalen algunos desafíos relacionados con la disposición de aforos (número de estudiantes, auxiliar de laboratorio, y docentes) para la correcta aplicación de los protocolos de bioseguridad, y las estrategias para verificación del estado de las muestras (especímenes depositados en colecciones de docencia). 


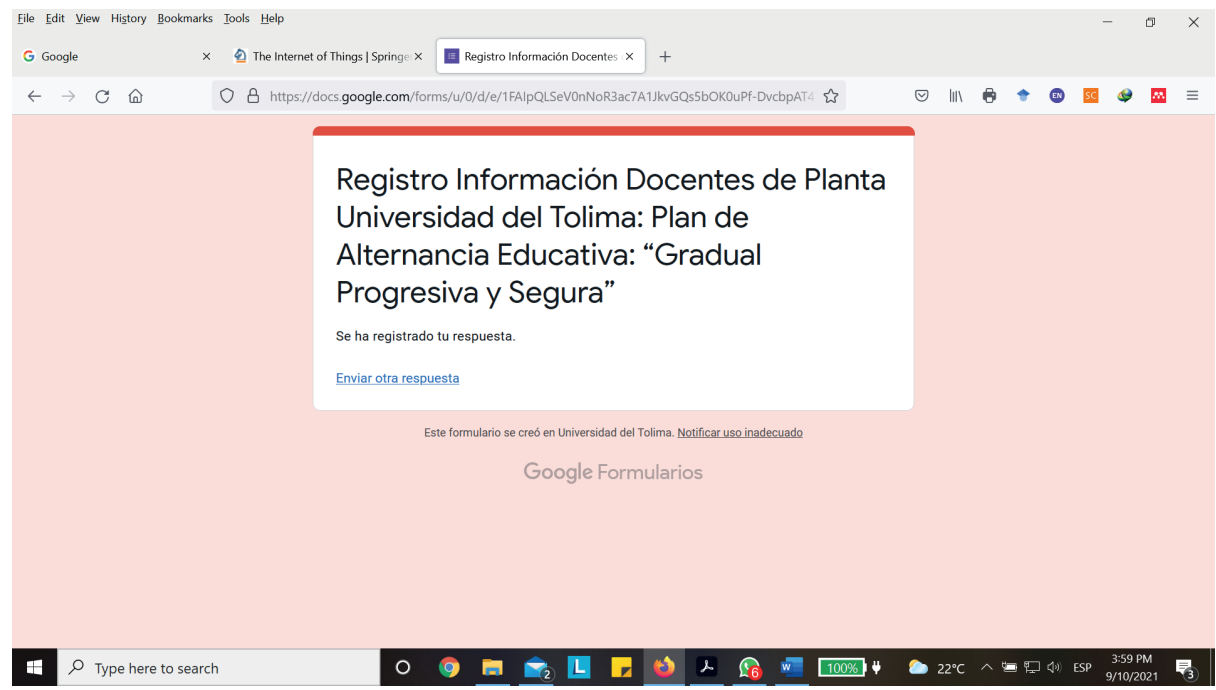

Figura 3. Ejemplo de registro final de uno de los formularios solicitados a los docentes por la Vicerrectoría Académica de la Universidad del Tolima, para retomar las actividades cotidianas en el campus central, a través de un Plan de Alternancia Educativa. Se propusieron formularios similares para los estudiantes. Este plan es soportado y actualizado permanentemente de acuerdo con la dinámica de la COVID-19, siguiendo las directrices del área de bienestar universitario que a su vez se apoya en los lineamientos del Ministerio de Salud y Protección Social de Colombia (https://www. minsalud.gov.co).

\section{Estrategias para el aseguramiento de la calidad}

Después de la declaratoria de pandemia, todos los sectores se vieron forzados a atender las normas establecidas por la OMS y políticas sanitarias propias de acuerdo con la dinámica de muertes, contagios y aforos en los centros de salud de cada país. Por lo tanto, las empresas, los centros laborales y las instituciones educativas en todos sus niveles tuvieron que reinventarse de acuerdo con las medidas implementadas (protocolos de bioseguridad), mientras hacía transición el acceso a alguna de las vacunas aprobadas. Se da entonces, la autorización para que, tanto las entidades públicas como privadas, reanuden sus actividades empleando sistemas de educación a distancia, los que estarán sujetos a la evaluación de los organismos competentes en el caso (Figueroa et al., 2021).

Nosotros discutimos algunos elementos que se pueden aplicar de manera amplia a otras carreras universitarias distintas a la biología, desde la perspectiva de los docentes. Sin embargo, el énfasis se realiza sobre los programas de Biología y Biología Ambiental de la UT y de la UI, respectivamente. Se trata de reconocer los elementos que propenden por el aseguramiento de la calidad y las oportunidades y desafíos de la pandemia bajo tres escenarios distintos (Figura 4). En el escenario 1 se resalta el aseguramiento de la calidad educativa avalada por las respectivas acreditaciones de calidad institucionales y/o de los programas, bajo una situación de normalidad académica antes de la pandemia. Es decir, actividades cotidianas presenciales sin restricción alguna, salvo las del día a día y sin una presión externa de naturaleza global. Allí se destaca un mayor esfuerzo del docente en sesiones magistrales, mayor participación en actividades prácticas de laboratorio y salidas de campo, y proporcionalmente un menor 
uso del computador e Internet. El escenario 2 ocurre bajo la declaratoria oficial de la pandemia, con varias restricciones marcadas por la cuarentena, el trabajo remoto o desde casa, cierre de fronteras, autoaislamiento y distanciamiento social, la esperanza de obtención de alguna dosis de vacuna, cierre de las IES y de sus laboratorios, un mayor uso y aplicación de las TIC con apoyo de la Internet (herramientas virtuales, capacitaciones en línea, redes sociales), limitación de la movilidad para el desarrollo de salidas de campo. No obstante, aquí se promueve el uso de investigaciones cualitativas o cuantitativas, aprovechando las ventajas de la información disponiblee en la nube o repositorios digitales. La Internet y los medios o redes sociales posibilitan la recogida de datos textuales, secuencias, imágenes o narrativas sobre una realidad limitada para el acceso a ella de forma presencial (Hernán-García et al., 2021). Bajo el escenario 3 , se establece, en principio, el inicio de las actividades educativas en alternancia (Ibarra-Caicedo, 2021b) y un retorno seguro, gradual y progresivo hacia las actividades cotidianas de presencialidad en los campus principales de las IES (Melo-Becerra et al., 2021). Se resalta la integración, incluso de manera proporcional, de actividades de campo, laboratorio y uso de la Internet, además de otras oportunidades para el manejo de la información recolectada con apoyo de las capacitaciones o experiencias recibidas en entornos virtuales, teletrabajo, teleeducación, entre otros (Figura 4).

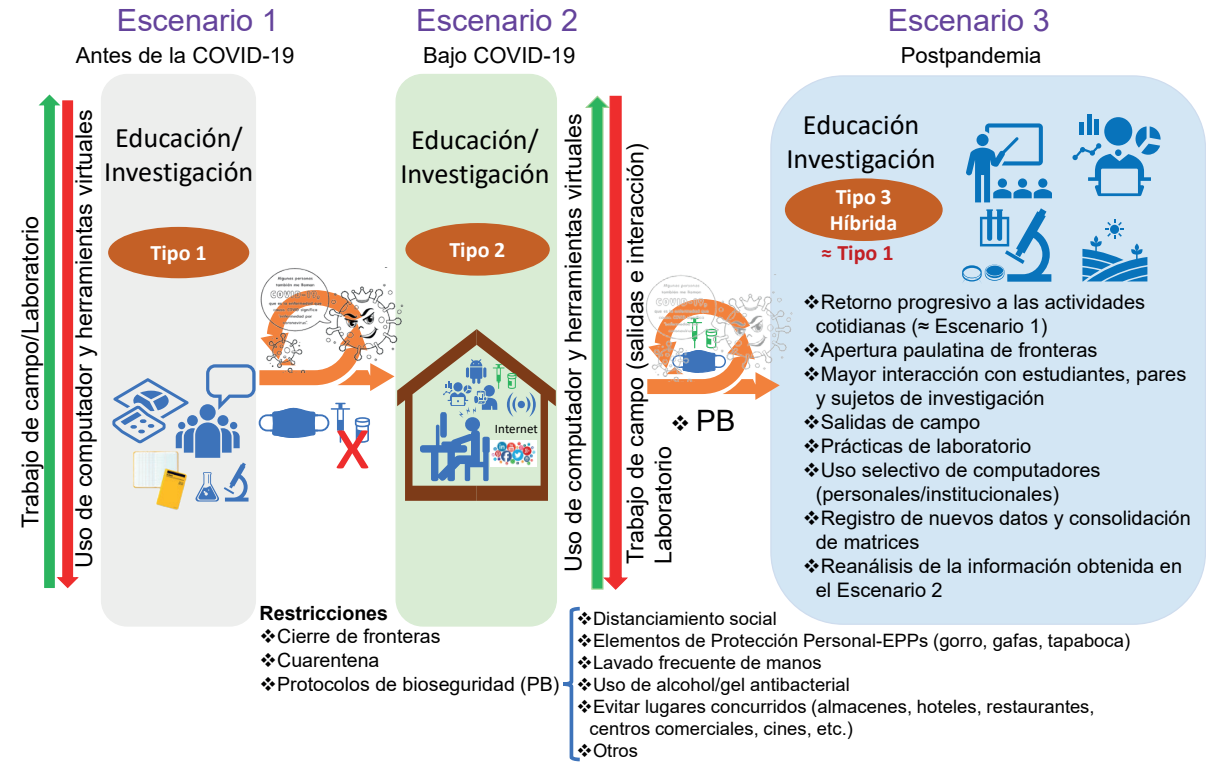

Figura 4. Escenarios de interacción para docentes y estudiantes de dos instituciones colombianas de educación superior frente a la dinámica de la pandemia por COVID-19, y los desafíos y oportunidades para el aseguramiento de la calidad. Se hace énfasis en las actividades educativas y de investigación en las carreras de pregrado de Biología y Biología Ambiental de la Universidad del Tolima y de la Universidad de Ibagué, respectivamente, asociadas con asignaturas de corte teórico-práctico (T/P), y T/P con salidas de campo. Las asignaturas de corte teóricas pueden aprovechar las ventajas del confinamiento (escenario 2), mientras que las T/P con salidas de campo se ven supeditadas al mantenimiento/levantamiento de las restricciones y cumplimiento de protocolos de bioseguridad (escenario 2), a la oportunidad de una transición bajo el modelo de alternancia (retorno gradual, progresivo y seguro a la presencialidad), y una posterior educación mixta, fortalecida durante la postpandemia (escenario 3). 
La dinámica de la pandemia confirmó la importancia de las TIC en el proceso de enseñanza en la educación universitaria tradicional; es decir, en aquellas IES con programas académicos presenciales. Además, el fortalecimiento de las competencias en el manejo de herramientas y entornos virtuales tanto de docentes como de estudiantes y administrativos durante las restricciones e interacciones desde casa, garantizan que éstos conocimientos sean puestos en práctica incluso después de la pandemia (escenario 3), puesto que es importante que los docentes sean conscientes del por qué, para qué y en qué momento utiliza las TIC en su labor, basados en teorías y enfoques que dan origen a metodologías y estrategias pedagógicas de trabajo dentro y fuera del aula (Arriaga et al., 2021). No obstante, bajo el contexto de la COVID-19, tanto en las instituciones públicas como privadas, se ha evidenciado una falta de condiciones mínimas o idóneas para continuar con el curso del ciclo escolar semestral, que operaba sin mayores traumatismos en presencialidad, cuando no se tenían las restricciones impuestas por la pandemia. En educación superior, las necesidades se evidencian con respecto a conocimientos más especializados, sujetos a exigencias sociales y demandas económicas (Miguel, 2020). Asimismo, es necesario profundizar en el sentido de conocer qué piensa la comunidad educativa: estudiantes, docentes y administrativos, con respecto a los obstáculos reales, los retos a los que se enfrentan y las competencias que están desarrollando o necesitan desarrollar para hacer frente a este contexto de pandemia y posteriormente (Miguel, 2020; Moral et al., 2021).

Los distintos escenarios (Figura 4), la brecha digital y la dinámica misma para un retorno a las labores cotidianas efectuadas bajo presencialidad, que se realizaban antes de la declaratoria de pandemia, ha afectado la planificación y desarrollo de la docencia, y la evaluación de resultados. Sin embargo, esta retroalimentación ha permitido comprobar que no toda la enseñanza, en programas con un alto componente práctico, se puede llevar a cabo de forma virtual. Algunos contenidos son susceptibles de virtualizar, otros requieren interacción, además de que se puedan virtualizar, y otros requieren necesariamente la presencia física (Núñez-Cortés, 2021).

\section{Enfoque STEAM+H: una alternativa en tiempos de COVID-19}

La resignificación de las universidades en su ser y actuar, a través de un nuevo estilo de organización para el futuro inmediato, debería surgir como un bálsamo de esperanza para la construcción colectiva en la educación, que necesita responder a los nuevos desafíos que afrontan los países, en medio de una crisis de la educación superior sin precedentes, profundizada por la pandemia (UNESCO, 2020).

Por lo tanto, este nuevo estilo basado en proyectos con enfoque STEAM+H (Ciencia, Tecnología, Ingeniería, Arte, Matemáticas e Humanidades; Science, Technology, Engineering, Arts, Mathematics \& Humanities, por sus siglas en inglés), representa una alternativa en tiempos de COVID-19 y de transformación digital (Fardoun et al., 2020). En un principio, surge el enfoque STEM durante la década de los noventa, de mano de la National Science Foundation (Ring et al., 2017), donde las nuevas metodologías se centraron en estas ramas del conocimiento, enfocadas al desarrollo de las competencias científico-tecnológicas. Sin embargo, este modelo de enseñanza-aprendizaje, fue el comienzo de un desarrollo que llevó a incluir las Artes (STEAM)-dado que son generadores de creatividad e innovación-y posteriormente las 
humanidades $($ STEAM $+\mathrm{H})$. El enfoque STEAM $+\mathrm{H}$ permite integrar los conocimientos desde diferentes disciplinas para cultivar un pensamiento y habilidades transformadoras, innovadoras y creativas para avanzar hacia un desarrollo sostenible y logro de la Agenda 2030, además de forjar habilidades que conviertan a los estudiantes en ciudadanos empoderados que participan en la atención de los problemas que hoy en día afectan a las localidades, los países y por supuesto las regiones (Skorton, 2019; UNESCO, 2019).

En este sentido, las universidades al asumir el enfoque de competencias (un concepto ampliado de "formación integral") como dimensión fundamental de su proyecto educativo institucional, pueden cultivar profundamente un alumnado, que utilice el enfoque STEAM+H, a través de un diálogo de saberes, en los que la institución se convierte en un actor que incorpora competencias con alta creatividad y gran habilidad para la innovación y resolución de problemas complejos. Aunque se reconoce que la educación STEAM+H es una propuesta pertinente para afrontar los procesos educativos en tiempos de la COVID 19, es necesario analizar el contexto, ya que no en todos se presentan las condiciones mínimas para su implementación (Vargas et al., 2020). Sin embargo, es necesario establecer un plan de acercamiento, para evaluar las situaciones sobre las cuales se podrían implementar estos proyectos. Este acercamiento sería mucho más masivo y probable bajo el actual panorama, que, aunque no es el mejor, si permite una transformación en la brecha digital a partir de la enseñanza remota de una nueva comunidad educativa en la postpandemia.

El enfoque de enseñanza para la educación STEAM+H, no solo permite un análisis y planificación curricular integral, sino que, a través de metodologías mixtas (sesiones sincrónicas/asincrónicas), el docente expone, orienta e interactúa con los estudiantes y, por otro lado, los participantes pueden retroalimentarse en foros, videos interactivos, plataformas comerciales o de acceso libre, con el fin de realizar prácticas que ayuden a consolidar su aprendizaje. De esta manera, los actores involucrados, pueden relacionarse en el marco de una comunidad de aprendizaje auténtica, como una característica emergente desde la transdisciplinariedad, aportando desde el saber y la experiencia, en la construcción colectiva de conocimiento, en la que los actores plantean soluciones a problemas complejos de la región, mediante la colaboración, gestión, creatividad y acción (Skorton, 2019). Por lo tanto, es posible integrar el STEAM+H en las aulas como parte de la educación por proyectos, formando continuadamente al profesorado de manera inter-y transdisciplinar (colaboración entre diferentes docentes de varias disciplinas), creando espacios físicos/virtuales y pedagógicos en los que se puedan desarrollar estos proyectos en beneficio de los estudiantes.

\section{Restricciones de la presente propuesta}

Nuestras vivencias pueden estar un poco sesgadas hacia las asignaturas de corte zoológico, que logramos desarrollar bajo la concepción teórico-práctica, y que a su vez, incluye al menos una salida de campo fuera de los espacios de las universidades seleccionadas, como se establece en su respectivo plan curricular. En ese sentido, el lector debe reconocer que se trata de una de las experiencias en el trabajo (enseñanza-aprendizaje) bajo interacción remota, con actividades y asignación de tareas tanto sincrónicas como asincrónicas, bajo una perspectiva futura de 
educación híbrida (presencial/virtual; Figura 4). Por lo tanto, se deben evaluar otros casos particulares donde también pueden existir muchas experiencias exitosas, dada la oportunidad de mejora y las mismas restricciones ocasionadas durante la pandemia por la COVID-19, que llevó a que los docentes tuvieran capacitaciones constantes con el ánimo de que mejoraran sus competencias en el manejo de herramientas virtuales, en el reconocimiento de distintas plataformas, y en la reinvención de su forma tradicional de enseñanza (Digión y Álvarez, 2021). Asimismo, generó en los estudiantes otras expectativas de compromiso, responsabilidad y seguimiento de las sesiones de interacción; por ejemplo, la ventaja de no poder asistir y contar con la grabación de la clase o discusión sobre alguna(s) sesión(es) pregrabada(s). Otro aspecto estuvo relacionado con la percepción sobre el proceso evaluativo desde ambas partes, el docente y los participantes.

En el mismo orden de ideas, se tiene que estos entornos virtuales de enseñanza permiten a través de sus plataformas de aprendizaje la comunicación del docente y el estudiante, bien sea en vivo o de forma asíncrona, empleando procedimientos asociados con TIC sin limitaciones de tiempo y espacio (Figueroa et al., 2021). Algunas de las opciones para considerar se encuentran en Vargas-Straube et al. (2021) para el caso de microbiología experimental, ecología acuática (https:// doi.org/10.24918/cs.2021.34), e invertebrados (Novo et al., 2021). No obstante, se deben tener presente las percepciones de los estudiantes con base en las nuevas alternativas de (auto)aprendizaje e interacción con el docente (Ortíz-Moreno et al., 2020; Urzúa et al., 2020; Vargas-Straube et al., 2021), al igual que las características de los programas y asignaturas (créditos prácticos); es decir, si estas últimas se imparten(ían) de manera regular o concentrada, con presencia de un elevado número de alumnos, y sobre todo las necesidades asociadas con la infraestructura y material de laboratorio para una adecuada realización del trabajo experimental (Argel et al., 2020; De la Sen y Díaz, 2009).

Finalmente, en asignaturas T/P durante la alternancia o retorno progresivo a la presencialidad, donde una parte de los estudiantes asisten al aula de clase, laboratorio o espacio abierto y la otra continúa bajo interacción remota, se debe reevaluar el uso de contenido, desde el punto de vista estático/dinámico; es decir, si se repite o modifica la misma práctica para cohortes subsiguientes. También se debe revisar el uso de muestras o especímenes en profundizaciones de disección externa e interna (ecomorfología), particularmente cuando es necesario mantener rotaciones en una práctica de laboratorio dependiendo del aforo (número máximo de usuarios de los equipos/ muestras disponibles) y del número inscritos y de asistentes de la asignatura. Desde nuestra experiencia, la educación presencial tradicional era relativamente estática en términos de contenidos, apropiación tecnológica (docentes de mayor edad versus los más jóvenes), y manejo de cohortes (misma asignatura, distintos estudiantes [salvo los repitentes]). Esta es, además, una excelente oportunidad para que los docentes más longevos reconozcan la realidad actual, sin calificar a priori un método de ineficaz, al tiempo que pueden reemplazar sus tradiciones y su tendencia a la repetición de fórmulas didácticas o pedagógicas del pasado (Prensky, 2001). En ese sentido, deben saber superar factores adversos como las costumbres, los hábitos comunales, la idiosincrasia, la cultura, entre otros. Sin embargo, el problema de la resistencia al 
cambio de paradigma sobre todo el de utilizar las TIC para el proceso de enseñanzaaprendizaje se mantiene latente en la actualidad en algunos colegas (Arriaga et al., 2021). Es importante también que se reconozcan las fortalezas y limitaciones, bajo el marco de lo que se ha discutido como "nativos e inmigrantes digitales", cuando se consideran a estudiantes y docentes, respectivamente (Guo et al., 2008; Jara y Prieto, 2018; Prensky, 2001). Se requiere entonces, promover la formación permanente de los profesores en el uso y en el manejo adecuado de TIC, lo cual permite la generación de modelos pedagógicos y estrategias de enseñanza más interactivas de acuerdo con las necesidades de los futuros profesionales (Jara y Prieto, 2018), que vieron limitadas sus competencias prácticas como consecuencia de las restricciones que nos impuso la pandemia por la COVID-19.

\section{Referencias}

Argel, N., Carasi, P., Manassero, C. y Quiroga, A. (2020). El trabajo experimental en tiempos de pandemia, desafíos y alternativas virtuales. $3^{\circ}$ Jornadas sobre las prácticas docentes en la Universidad Pública, 1-7. Recuperado de https://t.ly/ROtJ

Arriaga, W., Bautista, J. K. y Montenegro, L. (2021). Las TIC y su apoyo en la educación universitaria en tiempo de pandemia: una fundamentación facto-teórica. Conrado, 17(78), 201-206. http://scielo.sld.cu/pdf/rc/v17n78/1990-8644-rc-17-78-201.pdf

Barberá, E. y Badia, A. (2005). Hacia el aula virtual: actividades de enseñanza y aprendizaje en la red. Revista Iberoamericana de Educación, 36(9), 1-22. https://doi.org/10.35362/rie3692769

Bercheñi, V. y Mariño, S. (2021). Identificación de brechas digitales en pandemia: Experiencias en carreras de grado de Facultades de la Universidad Nacional del Nordeste. Argentina. SciComm Report, 1(2), 1-12. https://doi.org/10.32457/scr.v1i2.1444

Cevallos, G. E., Cedeño, E. R., Sánchez, V. B., Macas, K. M. y Ramos, Y. (2021). Educación en tiempos del COVID-19, perspectiva desde la socioformación. Dilemas contemporáneos: educación, política y valores, 8(spe1), 00004. https://doi.org/10.46377/dilemas.v8i.2558

Cipagauta, M. E. (2021). Pandemia, docencia y tecnología [pandemia; COVID-19; tecnología; redes académicas; profesores blogueros]. Revista Mexicana de Bachillerato a Distancia, 13(26), 1-4. https://doi.org/10.22201/cuaieed.20074751e.2021.26.80440

de la Sen, A. S. y Díaz, D. M. (2009). Diseño de prácticas virtuales para la enseñanza de Microbiológica Industrial. REDUCA (Biología). Serie Microbiología. Microbiología Industrial, 2(4), 35-57. Recuperado de https://bit.ly/3BCvt2z

Digión, L. B. y Álvarez, M. M. (2021). Experiencia de enseñanza-aprendizaje con aula virtual en el acompañamiento pedagógico debido al Covid-19. Apertura (Guadalajara, Jal.), 13(1), 20-35. https://doi.org/10.32870/ap.v13n1.1957

Fardoun, H., González, C., Collazos, C. A. y Yousef, M. (2020). Estudio exploratorio en Iberoamérica sobre procesos de enseñanza-aprendizaje y propuesta de evaluación en tiempos de pandemia. Education in the Knowledge Society (EKS), 21, 1-9. https://doi.org/10.14201/ eks.23537

Figueroa, J. J., Miranda, R. J., Alva, Y. M. y Miller, D. A. (2021). Retos de la educación universitaria virtual en Lima. Veritas Et Scientia, 10(1), 141-149. https://doi.org/10.47796/ves.v10i1.466

Gamage, K. A. A., Wijesuriya, D. I., Ekanayake, S. Y., Rennie, A. E. W., Lambert, C. G. \& Gunawardhana, N. (2020). Online Delivery of Teaching and Laboratory Practices: Continuity of University Programmes during COVID-19 Pandemic. Education Sciences, 10(10), 291. https://doi. org/10.3390/educsci10100291 
Giraldo, G. A., Gómez, M. M. y Giraldo, C. F. (2021). COVID-19 y uso de redes sociales virtuales en educación médica. Educación Médica, 22(5), 273-277. https://doi.org/10.1016/j. edumed.2021.05.007

Gómez-Arteta, I. y Escobar-Mamani, F. (2021). Educación virtual en tiempos de pandemia: incremento de la desigualdad social en el Perú. Chakiñan, Revista de Ciencias Sociales y Humanidades, 15, 152-165. https://doi.org/10.37135/chk.002.15.10

Guo, R. X., Dobson, T. y Petrina, S. (2008). Digital Natives, Digital Immigrants: An Analysis of Age and ICT Competency in Teacher Education. Journal of Educational Computing Research, 38(3), 235-254. https://doi.org/10.2190/EC.38.3.a

Hernán-García, M., Lineros-González, C. y Ruiz-Azarola, A. (2021). Cómo adaptar una investigación cualitativa a contextos de confinamiento. Gaceta Sanitaria, 35(3), 298-301. https://doi. org/10.1016/j.gaceta.2020.06.007

Ibarra-Caicedo, J. F. (2021a). B-learning como propuesta para la alternancia educativa. Revista Universitaria de Informática RUNIN, 8(11), 81-113. Recuperado de https://bit.ly/3H3ysST

Ibarra-Caicedo, J. F. (2021b). Pautas pedagógicas para la alternancia educativa. Boletín Informativo CEI, 8(1), 18-22. Recuperado de https://bit.ly/3H7kbom

Jandrić, P. (2020). Postdigital Research in the Time of Covid-19. Postdigital Science and Education, 2(2), 233-238. https://doi.org/10.1007/s42438-020-00113-8

Jandrić, P., Hayes, D., Truelove, I., Levinson, P., Mayo, P., Ryberg, T., ... \& Hayes, S. (2020). Teaching in the Age of Covid-19. Postdigital Science and Education, 2(3), 1069-1230. https://doi. org/10.1007/s42438-020-00169-6

Jara, N. P. y Prieto, C. (2018). Impacto de las diferencias entre nativos e inmigrantes digitales en la enseñanza en las ciencias de la salud: revisión sistemática. Revista Cubana de Información en Ciencias de la Salud, 29(1), 92-105. http://scielo.sld.cu/pdf/ics/v29n1/a7_1158.pdf

Melo-Becerra, L. A., Ramos-Forero, J. E., Rodríguez Arenas, J. L. y Zárate-Solano, H. M. (2021). Efecto de la pandemia sobre el sistema educativo: El caso de Colombia. Borradores de Economía, 1179, 1-58. Recuperado de https://bit.ly/3v0sDU1

Miguel, J. A. (2020). La educación superior en tiempos de pandemia: una visión desde dentro del proceso formativo. Revista Latinoamericana de Estudios Educativos, 50(Especial), 13-40. https://doi.org/10.48102/rlee.2020.50.ESPECIAL.95

Moral, R. R., Isturiz, J. S., García-Miguel, M. J., Cerro, A., de Leonardo, C. G., Sevilla, M. D. C. T., ... \& Salinas, I. (2021). Opiniones de profesores y alumnos sobre un programa integral online en medicina durante el confinamiento por COVID-19. Educación Médica, 22(4), 206-214. https://doi.org/10.1016/j.edumed.2021.01.011

Moreira, C. A., Hidalgo, W. A. y Martínez, R. M. (2020). E-learning y oficinas virtuales durante la pandemia del Covid-19. E-IDEA Journal of Business Sciences, 2(5), 8-15. Recuperado de https://bit.ly/3oZbzcX

Novo, M., Sánchez, N., Gutiérrez López, M., Cánovas, R. G., Pardos, F., Trigo, D. y Díaz Cosín, D. (2021). The lab In A box: A take-out practical experience for an online invertebrate biology course. Invertebrate Biology, 140(1), e12324. https://doi.org/10.1111/ivb.12324

Núñez-Cortés, J. M. (2021). Mitos y realidades de la enseñanza remota. Educación Médica, 22(6), 297. https://doi.org/10.1016/j.edumed.2021.12.001

Ordorika, I. (2020). Pandemia y educación superior. Revista de la educación superior, 49(194), 1-8. https://doi.org/10.36857/resu.2020.194.1120

Ortíz-Moreno, M. L., Guerrero, S. C. y Sandoval-Parra, K. X. (2020). Análisis de las expectativas didácticas estudiantiles para el aprendizaje virtual de la microbiología en la pandemia COVID-19. Memorias del Congreso Internacional de Ciencias Básicas e Ingeniería, Villavicencio, Colombia. Recuperado de https://bit.ly/3ld3k4l

Prensky, M. (2001). Digital Natives, Digital Immigrants Part 1. On the Horizon, 9(5), 1-6. https://doi. org/10.1108/10748120110424816 
Ring, E. A., Dare, E. A., Crotty, E. A. y Roehrig, G. H. (2017). The Evolution of Teacher Conceptions of STEM Education Throughout an Intensive Professional Development Experience. Journal of Science Teacher Education, 28(5), 444-467. https://doi.org/10.1080/104656 OX.2017.1356671

Ruiz-Aquino, M. (2020). El desafío de la presencialidad a la virtualidad en la educación superior en tiempos de pandemia. Desafios, 11(1), 7-8. https://doi.org/10.37711/desafios.2020.11.1.136

Santana-Sardi, G. A., Gutiérrez-Santana, J. A., Zambrano-Palacios, V. C. y Castro-Coello, R. L. (2020). La Educación Superior ecuatoriana en tiempo de la pandemia del Covid-19. Dominio de las Ciencias, 6(3), 757-775. https://doi.org/10.23857/dc.v6i3.1428

Skorton, D. (2019). Branches from the same tree: The case for integration in higher education. Proceedings of the National Academy of Sciences, 116(6), 1865. https://doi.org/10.1073/ pnas.1807201115

UNESCO (2019). UNESCO México presente en el segundo Foro Internacional Vanguardia en la Educación 2019. México. Recuperado de https://bit.ly/3s2BLWg

UNESCO (2020). Education: Education and Science as a Vaccine for the Pandemic. UNESCO. Recuperado de https://bit.ly/3v0TzTp

Urzúa, M., Rodríguez, D., Valencia, M. y Ruiz, R. (2020). Aprender ciencias experimentales mediante TIC en tiempos de covid-19: percepción del estudiantado. Praxis \& Saber, 11(27), e11447. https://doi.org/10.19053/22160159.v11.n27.2020.11447

Vargas-Straube, M. J., Gutiérrez, J. C., Soto, J. F. y Isla, E. (2021). Aprendizaje de microbiología experimental en un formato virtual y en contexto de pandemia: una experiencia de implementación de actividades con la utilización de un set portátil en la formación de profesores de Biología y Ciencias Naturales. Revista Iberoamericana de Educación, 87(1), 49-71. https://doi.org/10.35362/rie8714594

Vargas, J., Cuero, J. y Riveros, F. (2020). Transformación digital y enfoque STEAM, una alternativa en tiempos de COVID-19. Revista Espacios, 41(42), 326-334. https://doi.org/10.48082/ espacios-a20v41n42p28

Villela, F. y Contreras, D. S. (2021). La brecha digital como una nueva capa de vulnerabilidad que afecta el acceso a la educación en México. Academia y Virtualidad, 14(1), 169-187. https://doi.org/10.18359/ravi.5395

\section{Cómo citar en APA:}

Guevara, G., García-Melo, J. E. y Franco-Pérez, L. M. (2022). Oportunidades y desafíos de los docentes en programasde biología con asignaturas teórico-prácticas de dos instituciones colombianas de educación superior bajo restricciones de pandemia. Revista Iberoamericana de Educación, 88(1), 85-100. https://doi.org/10.35362/rie8814833 